\title{
HIGH PERFORMANCE PERFECT TRACKING CONTROL BASED ON MULTIRATE FEEDFORWARD / FEEDBACK CONTROLLERS WITH GENERALIZED SAMPLING PERIODS
}

\author{
Hiroshi Fujimoto* Yoichi Hori* Atsuo Kawamura ${ }^{* *}$ \\ * Department of Electrical Engineering, University of Tokyo, \\ Tokyo, 113-8656 Japan, email: fuji@hori.t.u-tokyo.ac.jp \\ ** Department of Electrical and Computer Engineering, \\ Yokohama National University, Yokohama, 240-8501 Japan
}

\begin{abstract}
In this paper, a novel perfect tracking control method based on the multirate sampling control is proposed, in which the concepts of the two-degreeof-freedom control are employed. Moreover, by generalizing the multirate input and output sampling periods, the proposed method can match the restrictions of the sampling periods caused by the hardware, and achieve higher performance. The main results of this paper are 1) the perfect tracking performance is assured in the intersampling points, if the input multiplicity is increased, 2) higher feedback performance is achieved, if the output multiplicity is increased. Illustrative examples of position control using a dc servo motor are presented, and simulations and experiments demonstrate the advantages of this approach. Copyright 1999 IFAC
\end{abstract}

Keywords: Digital control, motion control, multirate sampling control, two-degree-of-freedom, tracking control

\section{INTRODUCTION}

In the digital motion control system, either the point to point control or the tracking control is usually employed. The point to point controller moves the plant output from one point to another point without indicating the path. Thus, it sometimes has the large tracking error because the transient path is not concerned. In the tracking control, however, the controlled plant follows the smoothed desired trajectory. Therefore, the tracking controllers are employed for high speed and high precision servo systems.

The best tracking controller is ideally the Perfect Tracking Controller (PTC) which controls the controlled object with zero tracking error (Tomizuka, 1987). The perfect tracking can be achieved using the feedforward controller $C_{1}[z]$ which is realized by the inverse system of the closed-loop system $G_{c l}[z]$.

$$
C_{1}[z]=\frac{1}{z^{d} G_{c l}[z]}
$$

where $d$ is the relative degree of $G_{c l}[z]$.

However, the discrete-time plant discretized by the zero-order-hold usually has unstable zeros (Åström et al., 1984). Thus, $C_{1}[z]$ becomes unstable because $G_{c l}[z]$ has the unstable zeros. Therefore, in the conventional digital control systems utilizing the zero-order-holds, the perfect tracking is usually impossible.

From this viewpoint, two feedforward control methods are proposed for the discrete-time nonminimum phase plant by Tomizuka (1987). First, The Stable Pole Zero Canceling (SPZC) controller cancels all poles and stable zeros of the closedloop system, which has both phase and gain er- 
rors caused by the uncancellable unstable zeros. Second, Zero Phase Error Tracking Controller (ZPETC) adds the factors which cancel the phase error to SPZC. However, the gain error caused by the unstable zeros has remained.

A lot of methods are proposed to compensate the gain error of ZPETC (Haack and Tomizuka, 1991; Torfs et al., 1992; Gross et al., 1994). However, these methods are not able to realize the perfect tracking because the zero-order-holds are assumed to be employed.

Authors have proposed a novel perfect tracking control method in use of multirate feedforward control without utilizing the zero-order-hold (Fujimoto and Kawamura, 1998). In this paper, the feedback controller is also made multirate, and the periods of the plant input and output are generalized. Those generalizations improve the closed-loop characteristics and the tracking performance in the inter-sampling points.

Kabamba (1987) and Mita et al. (1990) resolved the unstable zeros problems of the discrete-time plant by zero assignment in use of multirate control. However, Moore et al. (1993) shows that those methods have disadvantages of large overshoot and oscillation in the inter-sampling points because the control input changes back and forth very quickly. On the other hand, the proposed method never has this problem because all of the plant states are controlled along the smoothed desired trajectories.

Recently, the modern sampled-data control theories have developed, which can optimize the intersample response (e.g. Chen and Francis, 1995; Hara et al., 1996). In contrast, the proposed method makes a simple and practical approach to grantee the smooth inter-sample responses by giving trajectories to all of the plant states (e.g. position and velocity) at every sampling point $T$.

\section{GENERALIZATIONS OF THE SAMPLING PERIODS}

A digital tracking control system usually has two samplers for the reference signal $r(t)$ and the output $y(t)$, and one holder on the input $u(t)$ as shown in Fig. 1. Therefore, there exist the three time periods $T, T_{y}$, and $T_{u}$ which represent the period of $r(t), y(t)$, and $u(t)$, respectively. The input period $T_{u}$ is generally decided by the speed of the actuator, D/A converter, or the calculation on the CPU. Moreover, the output period $T_{y}$ is also determined by the speed of the sensor or the $\mathrm{A} / \mathrm{D}$ converter.

Real control systems usually hold the restrictions on $T_{u}$ and/or $T_{y}$. Thus, the conventional digital

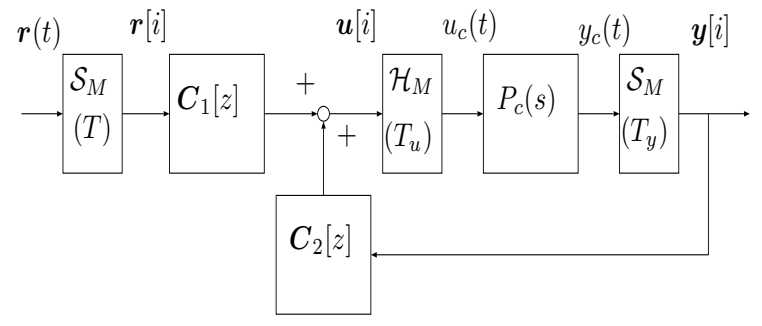

Fig. 1. Two-degree-of-freedom control system.

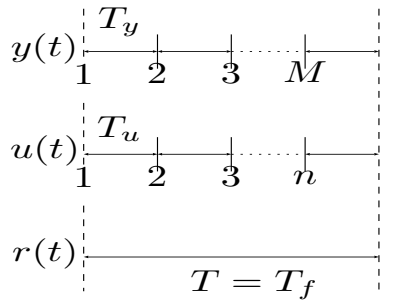

(a) $\operatorname{Case1}\left(T>T_{y}\right)$

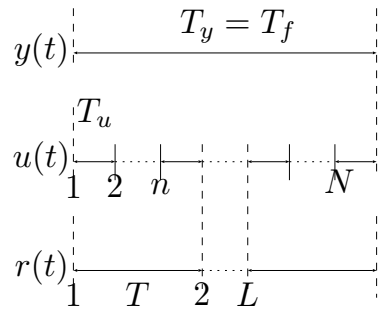

(b) Case2 $\left(T \leq T_{y}\right)$
Fig. 2. Multirate sampling control.

control systems make these three periods equal to the longer period between $T_{u}$ and $T_{y}$.

On the other hand, authors showed that the perfect tracking can be achieved on every sampling point $T$ by letting $T=n T_{u}$, where $n$ is the plant order (Fujimoto and Kawamura, 1998). Although the conventional methods with zero-orderhold are unable to realize the perfect tracking, the method has succeeded in it by introducing new control scheme. In spite of that, it has a problem; on the high order plant, the output sampling period $T_{y}$ gets too long and the closed-loop characteristics become poor because the assumption $T_{y}=n T_{u}$ is fixed.

In this paper, high performance perfect tracking control method is proposed, by which the control system matches the hardware restrictions on the periods of the plant input holding and output sampling. In the following discussions, $T=n T_{u}$ is regarded as the condition for the perfect tracking, and the assumption $T_{y}=T$ is removed and generalized.

In this paper, the following two cases are considered, which are very ordinary in industries. First, although $T_{u}$ is decided in advance by the hardware restrictions, the plant output can be detected $M$ times during $T\left(=n T_{u}>T_{y}\right)$, as shown in Fig. 2 (a). This case is referred to as case 1 in this paper. On this case, the closed-loop characteristics can be improved because much information of the plant can be detected. Second, although $T_{y}$ is decided in advance, the plant input can be changed $L \times n(\triangleq N)$ during $T_{y}$, as shown in Fig. 2(b). It is also referred to as case 2 . On this case, the perfect tracking can be assured $L$ times during inter- 


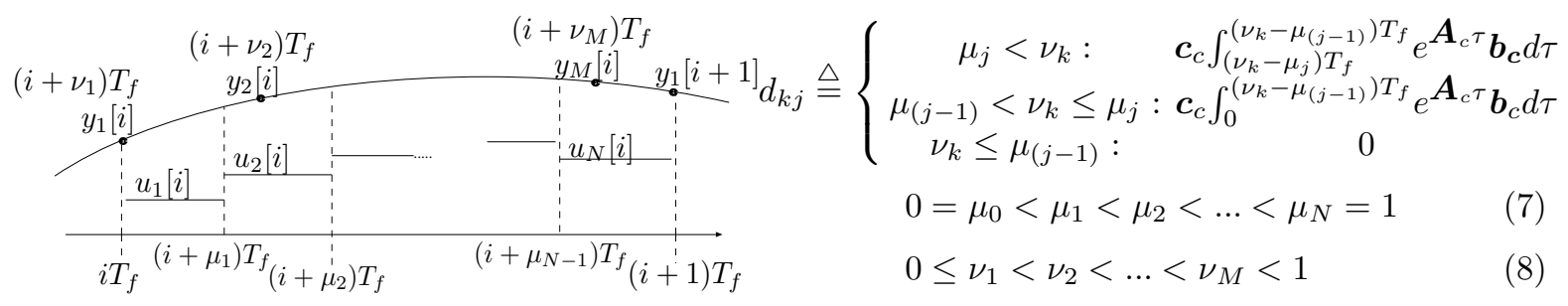

where $\mu_{j}(j=0,1, \cdots, N)$ and $\nu_{k}(k=1, \cdots, M)$

Fig. 3. Generalized multirate sampling control.

sample points of $T_{y}$. The output multiplicity $M$, input multiplicity $N$ and $L$ are positive integers.

In the two multi-period systems as shown in Fig. 2, the longer period between $T$ and $T_{y}$ is defined as the flame period $T_{f}$ (Araki, 1993). If this definition is employed, case 1 and 2 can be dealt with together.

\section{DESIGNS OF THE PROPOSED CONTROLLERS}

In this section, the proposed perfect tracking control method is presented. For simplification, the continuous-time plant is assumed to be SISO system. The proposed method, however, can be extended to deal with the MIMO system by the same way as Fujimoto et al. (1999).

\subsection{Plant Discretization and Parameterization}

In order to deal with case 1 and 2 together, the multirate control scheme is represented by Fig. 3 in use of input and output multiplicity $N$ and $M$. Thus, in case $1, N$ should be set to $n$. Moreover, in case $2, M$ also have to be set to 1 .

Consider the continuous-time $n$th order plant described by

$$
\dot{\boldsymbol{x}}(t)=\boldsymbol{A}_{c} \boldsymbol{x}(t)+\boldsymbol{b}_{c} u(t), \quad y(t)=\boldsymbol{c}_{c} \boldsymbol{x}(t) .(2)
$$

The discrete-time plant discretized by the generalized multirate sampling control (Fig. 3) becomes

$\boldsymbol{x}[i+1]=\boldsymbol{A} \boldsymbol{x}[i]+\boldsymbol{B} \boldsymbol{u}[i], \quad \boldsymbol{y}[i]=\boldsymbol{C} \boldsymbol{x}[i]+\boldsymbol{D} \boldsymbol{u}[i](3)$

where $\boldsymbol{x}[i]=\boldsymbol{x}(i T)$, and where matrices $\boldsymbol{A}, \boldsymbol{B}, \boldsymbol{C}, \boldsymbol{D}$ and vectors $\boldsymbol{u}, \boldsymbol{y}$ are given by

$$
\begin{gathered}
{\left[\begin{array}{c|c}
\boldsymbol{A} & \boldsymbol{B} \\
\hline \boldsymbol{C} & \boldsymbol{D}
\end{array}\right] \triangleq\left[\begin{array}{c|ccc}
e^{\boldsymbol{A}_{c} T_{f}} & \boldsymbol{b}_{1} & \cdots & \boldsymbol{b}_{N} \\
\hline \boldsymbol{c}_{1} & d_{11} & \cdots & d_{1 N} \\
\vdots & \vdots & & \vdots \\
\boldsymbol{c}_{M} & d_{M 1} & \cdots & d_{M N}
\end{array}\right]} \\
\boldsymbol{u} \triangleq\left[u_{1}, \cdots, u_{N}\right]^{T}, \boldsymbol{y} \triangleq\left[y_{1}, \cdots, y_{M}\right]^{T} \\
\boldsymbol{b}_{j} \triangleq \int_{\left(1-\mu_{j}\right) T_{f}}^{\left(1-\mu_{(j-1)}\right) T_{f}} e^{\boldsymbol{A}_{c} \tau} \boldsymbol{b}_{c} d \tau, \boldsymbol{c}_{k} \triangleq \boldsymbol{c}_{c} e^{\boldsymbol{A}_{c \nu_{k} T_{f}}}
\end{gathered}
$$

are the parameters for multirate sampling as shown in Fig. 3. If $T_{f}$ is divided at same intervals, $\mu_{j}=j / N, \nu_{k}=(k-1) / M$.

The coprime factorization of the pulse transfer function $\boldsymbol{P}[z]$ realized by (3) is described by

$$
\boldsymbol{P}[z]=\boldsymbol{N} \boldsymbol{M}^{-1}
$$

where $\boldsymbol{M}, \boldsymbol{N} \in \boldsymbol{R} \boldsymbol{H}_{\infty}$ (Sugie and Yoshikawa, 1986).

The proposed method employs the multirateinput control as the two-degree-of-freedom control, as shown in Fig. 1. In the figure, $\mathcal{H}_{M}, \mathcal{S}_{M}$ represent the multirate hold, the (multirate) sampler, respectively.

In the ideal tracking control system, the transfer characteristic $\left(G_{y r}\right)$ from the command $r$ to the output $y$ is generally 1 . In this paper, the feedforward controller $\boldsymbol{C}_{1}[z]$ is considered so that the transfer characteristic from the desired state $\boldsymbol{x}_{d}$ to the plant state $\boldsymbol{x}$ can be $\boldsymbol{I}$.

\subsection{Design of the feedback controller $\boldsymbol{C}_{2}[z]$}

Before the perfect tracking controller $\boldsymbol{C}_{1}[z]$ is designed, the $\boldsymbol{C}_{2}[z]$ has to be determined. Here, the given feedback controller $\boldsymbol{C}_{2}[z]$ must be a robust controller that let the sensitivity function $\boldsymbol{S}[z]=\left(\boldsymbol{I}-\boldsymbol{P}[z] \boldsymbol{C}_{2}[z]\right)^{-1}$ be small enough. The reason is that the sensitivity function $\boldsymbol{S}[z]$ represents the variation of the command response characteristic $\boldsymbol{G} \boldsymbol{y} \boldsymbol{r}$ under the variation of $\boldsymbol{P}[z]$ (Sugie and Yoshikawa, 1986).

In Fig. $1, \boldsymbol{C}_{2}[z]$ is described as a multirate input and multirate output controller. However, in this section, the simplest method is proposed, in which the $\boldsymbol{C}_{2}[z]$ is obtained from the popular single-rate controller such as a disturbance observer or $H_{\infty}$ controller.

First, in case $1, T_{y}$ is set to equal to $T_{u}(M=n)$, and $C_{2 s}\left[z^{\frac{1}{n}}\right]=\left\{\boldsymbol{A}_{s}, \boldsymbol{b}_{s}, \boldsymbol{c}_{s}, d_{s}\right\}$ is designed on sampling period $T_{y}$. Then, $\boldsymbol{C}_{2}[z]$ moving on every $T_{f}$ is obtained from $C_{2 s}\left[z^{\frac{1}{n}}\right]$ moving on every $T_{y}=T_{f} / n$ by (Chen and Francis, 1995)

$$
\boldsymbol{C}_{2}[z]=\left[\begin{array}{c|cccc}
\boldsymbol{A}_{s}^{n} & \boldsymbol{A}_{s}^{n-1} \boldsymbol{b}_{s} & \boldsymbol{A}_{s}^{n-2} \boldsymbol{b}_{s} & \cdots & \boldsymbol{b}_{s} \\
\hline \boldsymbol{c}_{s} & d_{s} & 0 & \cdots & 0 \\
\boldsymbol{c}_{s} \boldsymbol{A}_{s} & \boldsymbol{c}_{s} \boldsymbol{b}_{s} & d_{s} & \cdots & 0 \\
\vdots & \vdots & & & \vdots \\
\boldsymbol{c}_{s} \boldsymbol{A}_{s}^{n-1} & \boldsymbol{c}_{s} \boldsymbol{A}_{s}^{n-2} \boldsymbol{b}_{s} & \boldsymbol{c}_{s} \boldsymbol{A}_{s}^{n-3} \boldsymbol{b}_{s} & \cdots & d_{s}
\end{array}\right]
$$




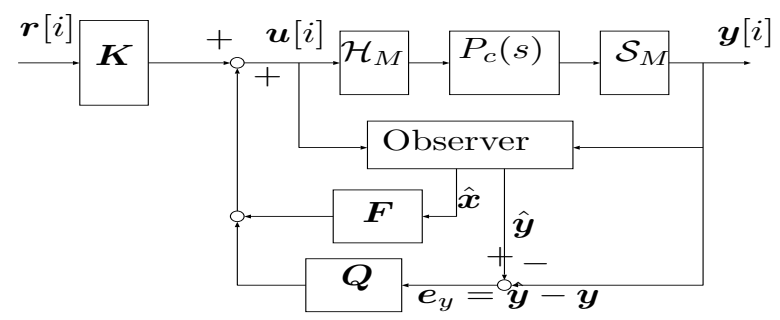

Fig. 4. Basic structure of TDOF control.

Second, in case $2, C_{2 s}[z]$ is designed on $T_{y}$. Then, the outputs of the $\boldsymbol{C}_{2}[z]$ become the same values during one sampling period $T_{y}$ as represented by

$$
C_{2}[z]=[\overbrace{C_{2 s}[z], \cdots, C_{2 s}[z]}^{N}]^{T} .
$$

\subsection{Design of the Perfect Tracking Controller $C_{1}[z]$}

In this section, the feedforward controller $\boldsymbol{C}_{1}[z]$ is designed so that the perfect tracking can be assured at every sampling point $T$.

(3) described on $T_{f}$ can be transferred to following equation on $T=T_{f} / L$.

$$
\overline{\boldsymbol{x}}[i+1]=\overline{\boldsymbol{A}} \boldsymbol{x}[i]+\overline{\boldsymbol{B}} \boldsymbol{u}[i]
$$

where $q \triangleq 1 / L$, and where matrices $\overline{\boldsymbol{A}}, \overline{\boldsymbol{B}}$ and vectors $\overline{\boldsymbol{x}}$ are given by

$$
\begin{aligned}
& \overline{\boldsymbol{x}}[i+1] \triangleq\left[\begin{array}{c}
\boldsymbol{x}[i+q] \\
\vdots \\
\boldsymbol{x}[i+l q] \\
\vdots \\
\boldsymbol{x}[i+1]
\end{array}\right], \quad \overline{\boldsymbol{A}} \triangleq\left[\begin{array}{c}
e^{\boldsymbol{A}_{\boldsymbol{c} T}} \\
\vdots \\
e^{\boldsymbol{A}_{\boldsymbol{c} l T}} \\
\vdots \\
e^{\boldsymbol{A}_{\boldsymbol{c} L T}}
\end{array}\right] \\
& \overline{\boldsymbol{B}} \triangleq\left[\begin{array}{cccccc}
\boldsymbol{B}_{L} & \boldsymbol{O} & \cdots & \cdots & \cdots & \boldsymbol{O} \\
\vdots & \ddots & & & \\
\boldsymbol{B}_{L-l} & \cdots & \boldsymbol{B}_{L} & \boldsymbol{O} & \ldots & \boldsymbol{O} \\
\vdots & & & \ddots & & \boldsymbol{O} \\
\boldsymbol{B}_{1} & \boldsymbol{B}_{2} & \cdots & \cdots & \cdots & \boldsymbol{B}_{L}
\end{array}\right] \\
& \boldsymbol{B}_{l}=\left[\boldsymbol{b}_{(l-1) n+1}, \cdots, \boldsymbol{b}_{l n}\right] \quad(l=1, \cdots, L) .
\end{aligned}
$$

From Fig. 1, the multirate control law of the proposed method is described by

$$
\begin{aligned}
\boldsymbol{u} & =\boldsymbol{C}_{1} \boldsymbol{r}+\boldsymbol{C}_{2} \boldsymbol{y} \\
& =\boldsymbol{F} \hat{\boldsymbol{x}}+\boldsymbol{Q} \boldsymbol{e}_{y}+\boldsymbol{K} \boldsymbol{r}
\end{aligned}
$$

where $\boldsymbol{K}, \boldsymbol{Q} \in \boldsymbol{R} \boldsymbol{H}_{\infty}$ are free parameters. Therefore, Fig. 1 can be transferred to Fig. 4 (Zhou et al., 1996). In this paper, $\boldsymbol{K}$ becomes a constant matrix.

Because the estimation errors of the observer become zero $\left(\hat{\boldsymbol{x}}=\boldsymbol{x}, \boldsymbol{e}_{y}=0\right)$ for the nominal plant, from (12) and (17), this system is represented by

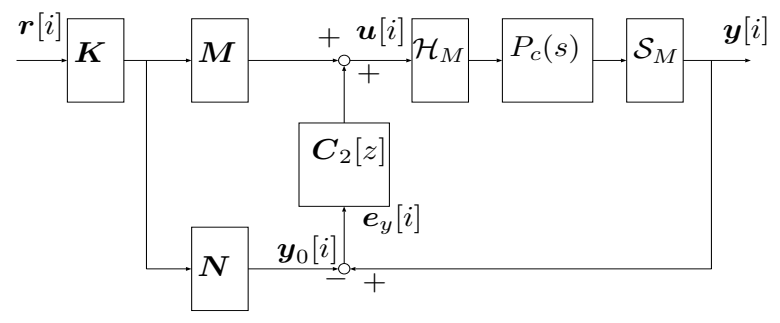

Fig. 5. Implementation of the proposed controller.

$$
\overline{\boldsymbol{x}}[i+1]=(\overline{\boldsymbol{A}}+\overline{\boldsymbol{B}} \boldsymbol{F}) \boldsymbol{x}[i]+\overline{\boldsymbol{B}} \boldsymbol{K} \boldsymbol{r}[i] .
$$

Because non-singularity of the matrix $\boldsymbol{B}$ can be assured by $T=n T_{u}$ (Araki, 1993), $\overline{\boldsymbol{B}}$ also becomes non-singular. Therefore, the parameters $\boldsymbol{F}, \boldsymbol{K}$ can be selected so that following equations are satisfied

$$
\bar{A}+\bar{B} \boldsymbol{F}=\boldsymbol{O}, \quad \bar{B} \boldsymbol{K}=\boldsymbol{I} .
$$

From (19), $\boldsymbol{F}, \boldsymbol{K}$ are given by

$$
\boldsymbol{F}=-\overline{\boldsymbol{B}}^{-1} \overline{\boldsymbol{A}}, \quad \boldsymbol{K}=\overline{\boldsymbol{B}}^{-1} .
$$

Therefore, (18) is described by

$$
\overline{\boldsymbol{x}}[i+1]=\boldsymbol{r}[i] .
$$

Utilizing the future desired state, let the reference input be

$$
\boldsymbol{r}[i]=\overline{\boldsymbol{x}}_{d}[i+1]
$$

where $\overline{\boldsymbol{x}}_{d}[i]$ is desired state. From (21) and (22), we find the perfect tracking $\overline{\boldsymbol{x}}[i]=\overline{\boldsymbol{x}}_{d}[i]$ can be achieved at every sampling point $T$.

Here, (16) and Fig. 1 can be transferred to (23) and Fig. 5 (Sugie and Yoshikawa, 1986). Therefore, the proposed controller can be simply implemented by substituting (23) for (20) and (24), and minimally realizing $\left[\boldsymbol{C}_{1}[z], \boldsymbol{C}_{2}[z]\right]$.

$$
\begin{gathered}
\boldsymbol{u}=\left(\boldsymbol{M}-\boldsymbol{C}_{2} \boldsymbol{N}\right) \boldsymbol{K} \boldsymbol{r}+\boldsymbol{C}_{2} \boldsymbol{y} \\
\boldsymbol{M}=\left[\begin{array}{c|c}
\boldsymbol{A}+\boldsymbol{B F} & \boldsymbol{B} \\
\hline \boldsymbol{F} & \boldsymbol{I}
\end{array}\right]=\boldsymbol{I}+z^{-1} \boldsymbol{F} \boldsymbol{B} \\
\boldsymbol{N}=\left[\begin{array}{ll}
\boldsymbol{A}+\boldsymbol{B F} & \boldsymbol{B} \\
\hline \boldsymbol{C}+\boldsymbol{D F} & \boldsymbol{D}
\end{array}\right]=\boldsymbol{D}+z^{-1}(\boldsymbol{C}+\boldsymbol{D F}) \boldsymbol{B}
\end{gathered}
$$

\section{ILLUSTRATIVE EXAMPLES}

In this section, the simulated and experimental results for the position tracking control system of the dc servo motor are presented, and the advantages of the proposed approach are demonstrated.

\subsection{Case2 on $L=1$}

First, the simplest examples in case 2 on $L=1$ are considered. The dc servo motor is described by

$$
P_{c}(s)=\frac{K}{J s^{2}} .
$$




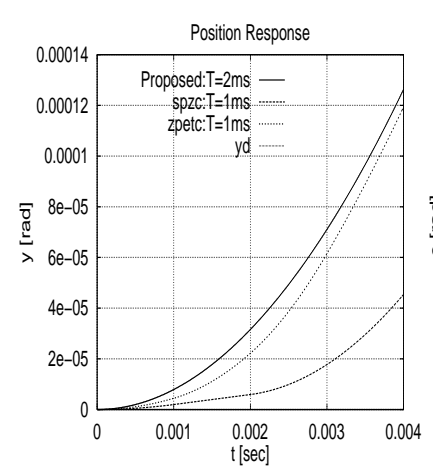

(a) Position

(Simulation)

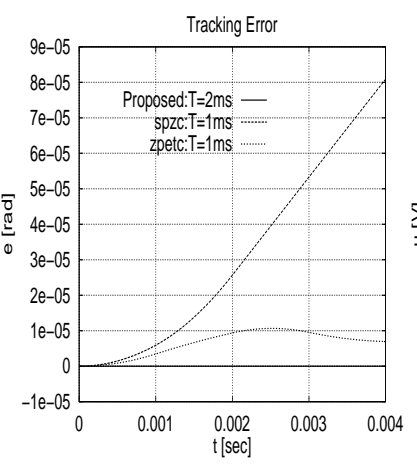

(b) Tracking error (Simulation)

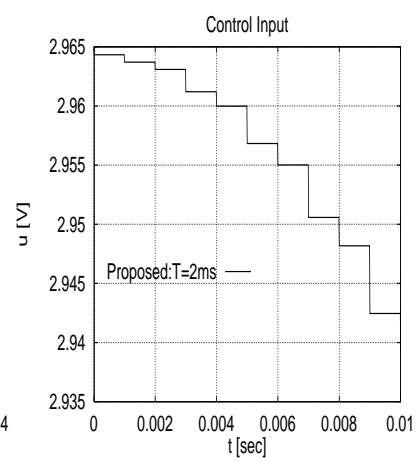

(c) Control input (Simulation)

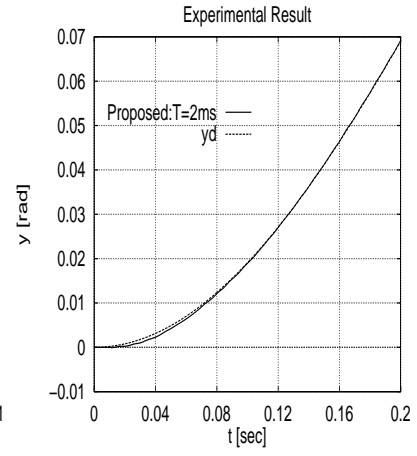

(d) Position (Experiment)

Fig. 6. Simulation and experimental results $\left(T_{r e f}=0.5[\mathrm{~s}]\right)$.

The feedback controller $C_{2}[z]$ is obtained from the $H_{\infty}$ mixed-sensitivity problem, which includes an integrator and becomes 3rd order(Mita et al., 1995). Calculating (23) and minimally realizing the obtained $\boldsymbol{C}_{1}[z]$ and $\boldsymbol{C}_{2}[z]$, the controller $\left[\boldsymbol{C}_{1}, \boldsymbol{C}_{2}\right]$ becomes 5 th order system.

Simulated and experimental results under the sinusoidal desired trajectories of period $T_{r e f}=$ $0.5[\mathrm{~s}]$ are shown in Fig. 6. Assuming $T_{y}=2[\mathrm{~ms}]$, the each periods become $T=2[\mathrm{~ms}]$ and $T_{u}=$ $T_{y} / L n=1[\mathrm{~ms}]$ because this plant is 2 nd order.

In the following simulations and experiment, the proposed method is compared with both SPZC and ZPETC proposed by Tomizuka (1987) at same input sampling period $\left(T_{u}\right)$. Therefore, the reference and the output sampling periods ( $T$ and $T_{y}$ ) of proposed method are twice as long as those of SPZC and ZPETC because of $T_{y}=$ $T_{u}=T=1[\mathrm{~ms}]$ on them. In spite of that, the results of proposed method have better tracking performance than those methods.

Fig. 6(a)(b) show that the proposed method gives better performance both than the SPZC and the ZPETC. While the responses of the SPZC and ZPETC have large tracking errors caused by the unstable zero, those of the proposed method have zero tracking errors. The simulated time response of the control input is shown in Fig. 6(c), which indicates that the control input of the proposed method is smooth in spite of using the multirate input control. Thus, we find the proposed multirate feedforward method is very practical. Moreover, the experimental result also indicates that the proposed method has high tracking performance as shown in Fig. 6(d). Furthermore, Fig. $6(\mathrm{a})$ (b) also show that the inter-sample responses are very smooth because not only position but also velocity follow the desired trajectories at every sampling point $T$.

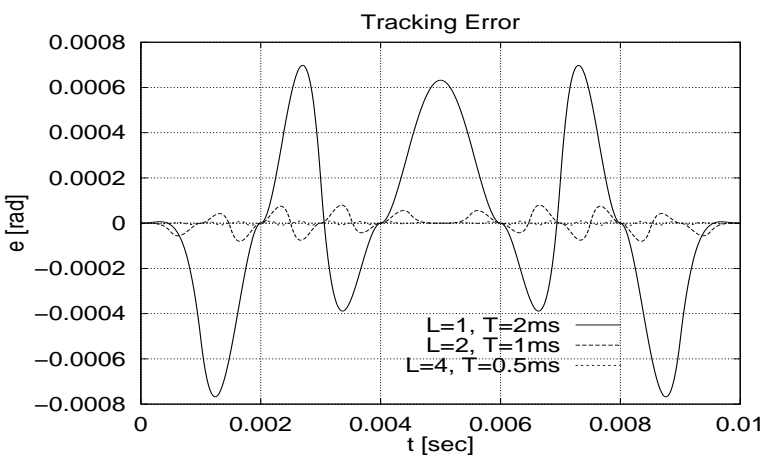

Fig. 7. Responses on $L=1,2,4\left(T_{\text {ref }}=10[\mathrm{~ms}]\right)$.

\subsection{Case2 on $L=2,4$}

Second, $T_{y}=2[\mathrm{~ms}]$ is fixed for the same plant as section 4.1, and the control input is changed more frequently than $T_{u}=1[\mathrm{~ms}]$.

Although in Fig. 6(b), the desired trajectories were very slow $\left(T_{r e f}=0.5[\mathrm{~s}]\right)$, Fig. 7 shows the simulated results for more fast trajectories $T_{\text {ref }}=$ $10[\mathrm{~ms}]$.

Compared with $L=1$, the tracking performances are improved for large input multiplicity $L=2,4$. The perfect tracking is also assured $L$ times during $T_{y}$.

\subsection{Case 1 on $M=n$}

Third, although $T_{y}=2[\mathrm{~ms}]$ is assumed in section 4.1, the output is detected more frequently at $T_{y}=T_{u}=1[\mathrm{~ms}]$ in this section. That is equal to case 1 on $M=n$.

In this case, the tracking performance is remained unchanged from section 4.1. However, the closed-loop characteristics can be improved because much information of the plant can be detected. Fig. 8 shows the simulated results when the plant inertia $J$ becomes three times larger than the nominal inertia $J_{n}$. We find that the 


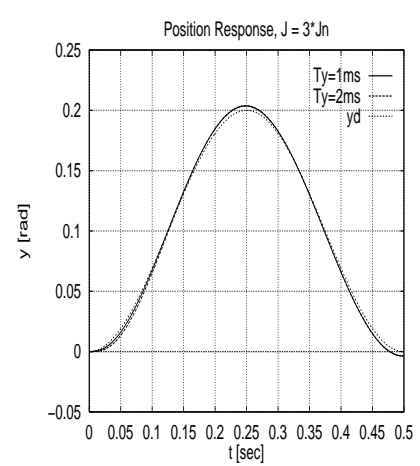

(a) Position

(Simulation)

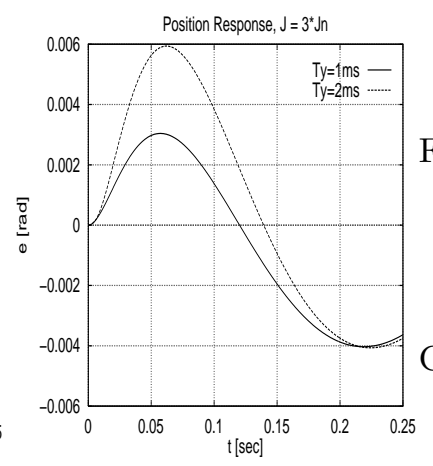

(b) Tracking error (Simulation)
Fig. 8. Responses under inertia variation $J=3 J_{n}$ $\left(T_{\text {ref }}=0.5[\mathrm{~s}]\right)$.

high performance robustness is achieved by the $H_{\infty}$ feedback controller as shown in Fig. 8(a). Moreover, Fig. 8(b) also shows that this case of $T_{y}=1[\mathrm{~ms}], M=2$ has higher performance robustness than the case of $T_{y}=2[\mathrm{~ms}], M=1$.

\section{CONCLUSION}

A novel perfect tracking control method in use of the multirate sampling control was proposed. Moreover, high performance perfect tracking control method was achieved, by which the control system matches the hardware restrictions on the periods of the plant input holding and output sampling. The remarkable advantages of the proposed method are 1) the tracking performance can be improved on the inter-sampling points, if the input can be changed more frequently, 2) higher feedback performance is achieved, if the output can be detected more frequently. Furthermore, illustrative examples of position control using a dc servo motor are performed, and the advantages of this approach are demonstrated.

Finally, the authors would like to note that part of this research is carried out with a subsidy of the Scientific Research Fund of the Ministry of Education.

\section{REFERENCES}

Araki, M. (1993). Recent developments in digital control theory. In: 12th IFAC World Congress. Vol. 9. pp. 251-260.

Åström, K. J., P. Hangander and J. Sternby (1984). Zeros of sampled system. Automatica 20(1), 31-38.

Chen, T. and B. Francis (1995). Optimal SampledData Control Systems. Springer.

Fujimoto, H., A. Kawamura and M. Tomizuka (1999). Generalized digital redesign method for linear feedback system based on N-delay control. IEEE/ASME Trans. Mechatronics. (to be published).

Fujimoto, H. and A. Kawamura (1998). Perfect tracking digital motion control based on twodegree-of-freedom multirate feedforward control. In: International Workshop on Advanced Motion Control. pp. 322-327.

Gross, E., M. Tomizuka and W. Messner (1994). Cancellation of discrete time unstable zeros by feedforward control. ASME Journal of Dynamics System, Measurement, and Control 116, 33-38.

Haack, B. and M. Tomizuka (1991). The effect of adding zeros to feedforward controllers. ASME Journal of Dynamics System, Measurement, and Control 113, 6-10.

Hara, S., Y. Yamamoto and H. Fujioka (1996). Modern and classical analysis / synthesis methods in sampled-data control - a brief overview with numerical examples -. In: Conf. on Decision and Control. pp. 12511256.

Kabamba, P. T. (1987). Control of linear systems using generalized sampled-data hold functions. IEEE Trans. Automat. Contr. 32(9), 772-783.

Mita, T., M. Hirata and S. B. Villas (1995). $H_{\infty}$ servo controller design for plants having poles on the $j \omega$ axis. In: Conf. on Decision and Control. pp. 2568-2573.

Mita, T., Y. Chida, Y. Kazu and H. Numasato (1990). Two-delay robust digital control and its applications - avoiding the problem on unstable limiting zeros. IEEE Trans. Automat. Contr. 35(8), 962-970.

Moore, K. L., S. P. Bhattacharyya and M. Dahleh (1993). Capabilities and limitations of multirate control schemes. Automatica 29(4), 941951.

Sugie, T. and T. Yoshikawa (1986). General solution of robust tracking problem in two-degreeof-freedom control systems. IEEE Trans. Automat. Contr. 31(6), 552-554.

Tomizuka, M. (1987). Zero phase error tracking algorithm for digital control. ASME Journal of Dynamics System, Measurement, and Control 109, 65-68.

Torfs, D., J. De Schutter and J. Swevers (1992). Extended bandwidth zero phase error tracking control of nonminimal phase systems. ASME Journal of Dynamics System, Measurement, and Control 114, 347-351.

Zhou, K., J. Doyle and K. Glover (1996). Robust and Optimal Control. Prentice-Hall, Inc. (Translated into Japanese by K.Z. Liu and Z.H. Luo). 